\title{
Observation of many-body dynamics in long-range tunneling after a quantum quench
}

\author{
Florian Meinert, ${ }^{1}$ Manfred J. Mark, ${ }^{1}$ Emil Kirilov, ${ }^{1}$ Katharina Lauber, ${ }^{1}$ \\ Philipp Weinmann, ${ }^{1}$ Michael Gröbner, ${ }^{1}$ Andrew J. Daley, ${ }^{2,3}$ \\ Hanns-Christoph Nägerl ${ }^{1 *}$ \\ ${ }^{1}$ Institut für Experimentalphysik und Zentrum für Quantenphysik, \\ Universität Innsbruck, 6020 Innsbruck, Austria \\ ${ }^{2}$ Department of Physics and Astronomy, University of Pittsburgh, Pittsburgh, PA 15260, USA \\ ${ }^{3}$ Department of Physics and SUPA, University of Strathclyde, Glasgow G4 0NG, UK \\ *To whom correspondence should be addressed; E-mail: Christoph.Naegerl@uibk.ac.at
}

Accepted version of Science Vol. 344 no. 6189, pp. 1259-1262 (2014)

DOI: $10.1126 /$ science. 1248402

Quantum tunneling constitutes one of the most fundamental processes in nature, and is at the heart of many quantum phenomena. We observe resonantlyenhanced long-range quantum tunneling in one-dimensional Mott-insulating Hubbard chains that are suddenly quenched into a tilted configuration. Higherorder tunneling processes over up to five lattice sites are observed as resonances in the number of doubly occupied sites when the tilt per site is tuned to integer fractions of the Mott gap. Second- and third-order tunneling is identified in the transient response after the tilt, from which we extract the characteristic scaling in accordance with perturbation theory and numerical simulations. This forms a basis for the controlled study of many-body dynamics 


\section{driven by higher-order tunneling, and demonstrates that when some degrees of freedom are frozen out, phenomena that are driven by small-amplitude tun- neling terms can still be observed, even when these amplitudes are lower than the total temperature of the system.}

Quantum tunneling is ubiquitous in physics and forms the basis for a multitude of fundamental effects (1) related to electronic transport, nuclear motion, light propagation, and superfluidity in lattice systems (2). While for weakly interacting particles tunneling at a rate $J$ will occur as an individual process for each particle, strong interactions change this situation completely, because the behaviour of each particle is then correlated with the behaviour of other particles. Such correlated tunneling processes are believed to play an important role, for example, in superconductivity of the celebrated cuprate systems (3-5). Second-order tunneling has been observed in cold atom experiments as driven resonances $(6)$ or directly as a dynamical process for pairs of strongly interacting particles in arrays of double well potentials (7). That process results in an effective nearest-neighbor super-exchange interaction $(8,9)$, which forms the basis of important forms of quantum magnetism (10), and provides a starting point for the formation of quantum many-body phases. Such tunneling processes have also recently been observed for electrons in systems of quantum dots (11).

Higher-order processes involving correlated tunneling across multiple lattice sites can give rise to longer-range effective interaction terms, and more complex many-body critical phenomena (12). Parallels can been drawn between long-range tunneling processes in tilted lattices and multi-photon electron-positron creation in strong electric fields, with connections to relativistic phenomena such as the Sauter-Schwinger effect in tilted Mott insulators (13), and also to longdistance electron transport in e.g. molecular systems $(14,15)$. However, while single-particle tunneling loss via higher band resonances (16) has been demonstrated, it has been difficult to observe coherent quantum dynamics due to higher-order tunneling processes, because the 
amplitude driving these terms is much smaller than the energy scale of temperatures for cold atoms.

Here, we directly observe dynamics in a quantum many-body system dominated by secondand third-order tunneling and identify resonant fourth- and fifth-order tunneling processes. We study long-range many-body tunneling processes that we resonantly enhance in tilted onedimensional (1D) Mott-insulating "Ising" chains of bosons (17-21). Specifically, we perform a quantum quench to a highly non-equilibrium situation by rapidly tilting a one-atom Mott insulator to an integer fraction $U / n$ of the Mott gap $U$. We record the probability for atom-pair formation as a function of time after the quench and find that the rate for pair formation is set by $\alpha_{n} \times J^{n} /(U / n)^{n-1}$ for $n=2$ and 3 with $\alpha_{n} \approx 36$ in accordance with the expected scaling $\propto J^{n} / U^{n-1}$ from perturbation theory. As we discuss in the supplementary material (22), the quench onto a critical point in the many-body system results in many-body dephasing of oscillations in the atom-pair number, corresponding to a characteristic growth in many-body entanglement (23-25) in our numerical simulations.

Our experiment is based on an array of 1D chains of atoms in an optical lattice near zero temperature (20). We model the system by a single-band Bose-Hubbard (BH) Hamiltonian $(22,26)$. For $U \gg J$ the many-body ground state is a Mott insulator with unit occupation at commensurate filling (Fig.1 A). This phase is characterized by exponentially localized atoms and highly suppressed tunneling. In addition, we superimpose a linear gradient potential, which introduces a site-to-site constant energy shift $E$. Tilting the initial Mott state quickly to $E \approx$ $U / n$ initiates resonant tunneling to the $n$-th neighbor for all sites simultaneously (lower part of Fig.1 A). For $n=1$ one couples to nearest-neighbor dipole states and observes strong coherent oscillations in the number of doubly occupied sites (doublons) with a characteristic frequency $4 J$ (20). For $n>1$ resonant tunnel coupling occurs across $n-1$ intermediate lattice sites. The process involves up to $n$ other particles, giving rise to occupation-dependent $n$-th-order 
tunneling. Since all particles participate in a tunnel process across $n$ sites, one expects the build-up of massive correlations in the interacting many-body system.

We prepare an ensemble of 1D Mott insulators (20) starting from a 3D Bose-Einstein condensate $(\mathrm{BEC})$ of typically $8.5 \times 10^{4} \mathrm{Cs}$ atoms without detectable uncondensed fraction. The BEC is levitated against gravity by a magnetic field gradient of $|\nabla B| \approx 31.1 \mathrm{G} / \mathrm{cm}$ and initially held in a crossed optical dipole trap $(20,27)$. We load the sample adiabatically into a cubic 3D optical lattice generated by laser beams at a wavelength of $\lambda_{1}=1064.5 \mathrm{~nm}$, thereby creating a singly-occupied 3D Mott insulator for a lattice depth of $V_{q}=20 E_{\mathrm{R}}(28)$ in each direction $(q=x, y, z)$ with less than $4 \%$ residual double occupancy. Here, $E_{\mathrm{R}}=\hbar^{2} k_{1}^{2} /(2 m)$ is the photon-recoil energy, with $k_{1}=2 \pi / \lambda_{1}$ and $m$ the mass of the Cs atom. The optical lattice results in a residual harmonic confinement of $\nu_{z}=11.9(2) \mathrm{Hz}$ in the $z$-direction of gravity. A broad Feshbach resonance allows us to set the atomic scattering length $a_{\mathrm{s}}$ and thus $U$ independently of $J$ by means of an offset magnetic field $B(20)$.

Tunneling resonances are observed by quickly tilting the lattice in the $z$-direction through a reduction of $|\nabla B|$ and then lowering $V_{z}$ to $10 E_{\mathrm{R}}$ within $1 \mathrm{~ms}$, giving $J \approx 25 \mathrm{~Hz}(26,28)$. All dynamics is now restricted along 1D Mott chains with an average length of 40 sites (20). The chains, in total $\approx 2000$, are decoupled from each other on the relevant experimental timescales. We let the systems evolve for a hold time $t_{\mathrm{h}}$ of up to $200 \mathrm{~ms}$ in the tilted configuration and then quickly ramp back $V_{z}$ to its original value and remove the tilt. The ensemble is characterized by measuring the number of doubly occupied sites $N_{\mathrm{d}}$ through Feshbach molecule formation with an overall efficiency of $80(3) \%(20)$. Alternatively, we detect the emergence of momentum-space coherence in time-of-flight (TOF) by quickly turning off all trapping potentials and allowing for $20 \mathrm{~ms}$ of free levitated expansion at $a_{\mathrm{s}}=0$ (27) before taking an absorption image.

The experimental result for a specific choice of $U=1077(20) \mathrm{Hz}$ is shown in Fig. 1 B. 
For a hold time of $t_{\mathrm{h}}=200 \mathrm{~ms}$ the transient response as discussed below has settled to a steady-state value. Besides a broad resonance at $E=1095(2) \mathrm{Hz}$ with FWHM $=172(9) \mathrm{Hz}$, two narrower resonances at $E=532(1)$ and $351(1) \mathrm{Hz}$ with $\mathrm{FWHM}=44(2)$ and $27(2) \mathrm{Hz}$ can be seen. While the broad resonance is the result of resonant tunnel coupling to nearestneighbor dipole states at $E_{1}=U(20)$, the positions of the narrower resonances are consistent with $E_{2}=U / 2$ and $E_{3}=U / 3$ and we hence interpret them to emerge from tunnel processes extending over a distance of two and three lattice sites, respectively. The reduced widths reflect the smaller amplitude of the higher-order tunnel processes. We believe that the resonances are slightly broadened inhomogeneously by the external harmonic confinement. The assignment of the resonance features to tunneling processes over multiple lattice sites is supported by TOF images (insets to Fig. $1 \mathrm{~B}$ ) taken for each resonance $E_{n}$ in the course of the transient response. The images clearly exhibit matter-wave interference patterns, indicating delocalization of the atoms during the tunnel processes. The integrated line densities are presented in Figs. 1 (C)(E). The periodicity of the sinusoidal density modulation, determined to $2 \hbar k_{1} / n$, is in agreement with spatial coherence of the atomic wave function over a distance of $n$ sites.

We now investigate the transient dynamics following the quantum quench. Fig. $2 \mathrm{~A}$ and $\mathrm{C}$ (B and D) show the on-resonance response of $N_{\mathrm{d}}$ and the fringe visibility $V$ in the TOF images for $E_{1}\left(E_{2}\right)$. The quench to $E_{1}$ results in large amplitude oscillations for $N_{\mathrm{d}}$, which decay on a timescale of a few tens of ms. The dynamics for $E_{2}$ are highly overdamped and fit to a saturated growth function of the form $\propto\left(1-e^{-t_{\mathrm{h}} / \tau}\right)$ with a characteristic rate $1 / \tau$. Interestingly, on both resonances $N_{\mathrm{d}}$ relaxes to the same stationary value. The oscillations for $E_{1}$ at frequency $4 J$ have been topic of our study in Ref. (20). The measured decay rates for the oscillatory response in $N_{d}$ are consistent with the width of the resonance in Fig. 1 B. Calculations show that the decay is due to many-body dephasing, which plays an increased role for larger chain lengths (20). The oscillatory response at $E_{1}$ is clearly reflected in the dynamics for $V$, as each 
local minimum coincides with an extremum for $N_{\mathrm{d}}$. For $E_{2}$ a simple three-site $\mathrm{BH}$ model predicts oscillations at frequency $\nu_{2}=4(2 \sqrt{2}+\sqrt{2}) J^{2} / U$ (22). In the experiment we find a single maximum for $V$ before it decays. The dephasing here results from more complicated dynamics in BH chains longer than three sites. In that case multiple atoms can tunnel, and interparticle interactions give rise both to constraints on which atoms can tunnel simultaneously and to modifications of the tunneling amplitude (22). This results in processes with many competing frequency components, dephasing the oscillations in the doublon number. While the dephasing can be enhanced by inhomogeneities, e.g., due to the harmonic confinement, such contributions are small relative to the intrinsic dephasing in longer chains (22).

We now focus on the scaling of the resonant doublon growth rate $1 / \tau$ with $J$ and $U$ for the resonance $E_{2}$. Example data sets, shown in Fig. 3 A, clearly demonstrate that $1 / \tau$ depends not only on $V_{z}$ alone, but also on $U$ when $V_{z}$ and thereby $J$ is kept constant. In Fig. 3 B we plot the same data with the time axis rescaled by the energy scale $J^{2} /(U / 2)$ for a second-order tunneling process. Remarkably, the data collapses onto a single curve, demonstrating that indeed secondorder tunneling dominates the transient dynamics following the quench. The experimental data is supported by numerical simulations for 10-30 site BH chains (22). The numerical data shows the same rise characteristics and reveals the same scaling collapse, see Fig. 3 C. Next, we extract values for $1 / \tau$ from measurements taken at different combinations of $V_{z}$ and $U$ and plot them in Fig. $3 \mathrm{D}$ as a function of $J^{2} /(U / 2)$. Our data reveals a linear dependence with a surprisingly large prefactor $\alpha_{2}=38(2)$, which we analyze in two ways. First, we compare to the frequency of coherent doublon oscillations in the simple three-site model. The role of many-body dephasing faster than a full second-order tunneling cycle is estimated by assuming $\tau$ as a quarter of the full tunneling period. The value $1 / \tau \approx 4 \times \nu_{2}$ is indicated by the solid line in Fig. 3 D. Second, we extract a characteristic growth rate from the numerical data, indicated by the dashed regions in Figs. 3 (C) and (D), revealing very good quantitative agreement with 
the experiment.

Along the same lines we investigate the dynamical scaling of the resonant response at the resonance $E_{3}=U / 3$. The dynamics that we observe for different combinations of $J$ and $U$ (Fig. $3 \mathrm{E}$ ) is qualitatively very similar to the one at $E_{2}$. The doublon number $N_{\mathrm{d}}$ settles to a steady-state value over a characteristic time $\tau$. In view of a third-order tunneling process we rescale the time axis by $J^{3} /(U / 3)^{2}$ (Fig. 3 F). Again we find a striking collapse of the data. The result of our numerics is shown in Fig. 3 G. Also the numerical data collapses onto a single curve. Residual oscillations after the initial growth period relate to the finite system size and the lack of averaging over positions in the trap (22). In Fig. $3 \mathrm{H}$ we plot $1 / \tau$ as a function of $J^{3} /(U / 3)^{2}$. Again the data collapses onto a single line. From the linear fit we obtain a slope of $\alpha_{3}=34(2)$. This is in good agreement with a characteristic growth rate determined from the numerical data, which we indicate by the dashed region as before. We note that the signature of the third-order process is not spoiled by the presence of second-order energy shifts (22).

Our data raises the question to what extent one can reverse the many-body dephasing dynamics. In Fig. 4 A we show the result of a many-body echo experiment for which we switch the sign of $U$ and $E$ at the $E_{2}=U / 2$ resonance in the course of the transient response. A clear, although only partial reversal in the time evolution for $N_{\mathrm{d}}$ can be seen before $N_{\mathrm{d}}$ raises to the same steady-state value as before. It would be interesting to test whether the revival could be improved by also a switching the sign of $J$. Naively, the second-order process scaling with $J^{2}$ should not depend on the sign of $J$. Switching $J$ by means of modulation techniques $(30)$ shall allow a detailed benchmarking of many-body damping versus the presence of mere inhomogeneous broadening in our system.

Finally, in Fig. 4 B we show resonances corresponding to many-body tunneling across four and five lattice sites. For this data the lattice depth was reduced to $V_{z}=7 E_{\mathrm{R}}$ to speed up the processes while still assuring that the systems are initially in the Mott-insulating regime. 
With decreasing $V_{z}$ the resonances at $U / 2$ and $U / 3$ slightly broaden, which we attribute to the increase of the second- and third-order tunneling rate. The new resonances at $U / 4$ and $U / 5$ are clearly detectable. We note that these fourth and fifth order tunneling processes greatly benefit from substantial Bose enhancement (22) and speculate that even higher-order processes should become accessible when one eliminates residual parabolic energy shifts due to the trapping laser beams.

Our results underline the utility of cold atoms in optical lattices for the investigation of fundamental physical processes driven by small-amplitude terms and specifically higher-order tunneling. By partly freezing the motion in the deep lattice, these sensitive processes can be observed here despite finite initial temperatures (which lead to defects and missing atoms). This will motivate further investigation of quantum phases and critical properties near these higherorder resonances, which are presently unknown, including systems with tilts along multiple axes $(19,31)$. Our initial studies of parameter reversals also open the door to the study of many-body dephasing and echo-type experiments on a quantum many-body system, as well as investigations into the nature of the many-body dephasing and (apparent) thermalization (32). Parallels can be drawn with arrays of quantum dots, opening further possibilities to model electron tunneling over multiple sites (11) by using fermionic atoms.

\section{References and Notes}

1. J. Ankerhold, Quantum Tunneling in Complex Systems. The Semiclassical Approach (Springer, Berlin, 2010).

2. O. Morsch and M. Oberthaler, Rev. Mod. Phys. 78, 179 (2006).

3. A. H. MacDonald, S. M. Girvin, and D. Yoshioka, Phys. Rev. B 37, 9753 (1988).

4. P. W. Anderson et al., J. Phys. Condens. Matter 16, R755 (2004). 
5. M. R. Norman, Science 332, 196 (2011).

6. R. Ma, M. E. Tai, P. M. Preiss, W. S. Bakr, J. Simon, and M. Greiner, Phys. Rev. Lett. 107, 095301 (2011).

7. S. Fölling, S. Trotzky, P. Cheinet, M. Feld, R. Saers, A. Widera, T. Müller, and I. Bloch, Nature 448, 1029 (2007).

8. S. Trotzky, P. Cheinet, S. Fölling, M. Feld, U. Schnorrberger, A. M. Rey, A. Polkovnikov, E. A. Demler, M. D. Lukin, and I. Bloch, Science 319, 295 (2008).

9. D. Greif, T. Uehlinger, G. Jotzu, L. Tarruell, and T. Esslinger, Science 340, 1307 (2013).

10. E. Dagotto, Rev. Mod. Phys. 66, 763 (1994).

11. F. R. Braakman, P. Barthelemy, C. Reichl, W. Wegscheider, and L. M. K. Vandersypen, Nature Nanotech. 8, 432 (2013).

12. S. Sachdev, Nature Physics 4, 173 (2008).

13. F. Queisser, P. Navez, and R. Schützhold, Phys. Rev. A 85, 033625 (2012).

14. G. Sedghi et al., Nature Nanotech. 6, 517 (2011).

15. J. R. Winkler and H. B. Gray, J. Am. Chem. Soc. 136, 2930 (2014).

16. C. Sias, A. Zenesini, H. Lignier, S. Wimberger, D. Ciampini, O. Morsch, and E. Arimondo, Phys. Rev. Lett. 98, 120403 (2007).

17. J. Simon, W. S. Bakr, R. Ma, M. E. Tai, P. M. Preiss, and M. Greiner, Nature 472, 307 (2011). 
18. W. S. Bakr, P. M. Preiss, M. E. Tai, R. Ma, J. Simon, M. Greiner, Nature 480, 500 (2011).

19. S. Sachdev, K. Sengupta, and S. M. Girvin, Phys. Rev. B 66, 075128 (2002).

20. F. Meinert, M. J. Mark, E. Kirilov, K. Lauber, P. Weinmann, A. J. Daley, and H.-C. Nägerl, Phys. Rev. Lett. 111, 053003 (2013).

21. M. Kolodrubetz, D. Pekker, B. K. Clark, and K. Sengupta, Phys. Rev. B 85, 100505 (2012).

22. Materials, methods, and additional theoretical background are available as supporting material on Science Online.

23. L. Amico, R. Fazio, A. Osterloh, and V. Vedral, Rev. Mod. Phys. 80, 517 (2008).

24. S. Trotzky, Y-A. Chen, A. Flesch, I. P. McCulloch, U. Schollwöck, J. Eisert, and I. Bloch, Nature Physics 8, 325 (2012).

25. C. Kollath, A. M. Läuchli, and E. Altman, Phys. Rev. Lett. 98, 180601 (2007).

26. D. Jaksch, C. Bruder, J.I. Cirac, C.W. Gardiner, and P. Zoller, Phys. Rev. Lett. 81, 3108 (1998).

27. T. Weber, J. Herbig, M. Mark, H.-C. Nägerl, and R. Grimm, Science 299, 232 (2002).

28. The lattice depth $V_{q}$ is calibrated via Kapitza-Dirac diffraction. The statistical error for $V_{q}$ is $1 \%$, though the systematic error can reach up to $5 \%$. We give all energies in frequency units. 
29. The matter-wave interference pattern is fit by $n(p)=$ $N_{0} e^{-\left(p-p_{0}\right)^{2} / w^{2}}\left(1+V \cos \left[k\left(p-p_{0}\right)+\phi\right]\right)$ with the fringe visibility $V$, the wave vector $k$, and a phase $\phi$.

30. H. Lignier, C. Sias, D. Ciampini, Y. Singh, A. Zenesini, O. Morsch, and E. Arimondo, Phys. Rev. Lett. 99, 220403 (2007).

31. S. Pielawa, T. Kitagawa, E. Berg, and S. Sachdev, Phys. Rev. B 83, 205135 (2011).

32. M. Rigol, V. Dunjko, and M. Olshanii, Nature 452, 854 (2008).

33. We are indebted to R. Grimm for generous support, and thank J. Schachenmayer for discussions and contributions to numerical code development. We gratefully acknowledge funding by the European Research Council (ERC) under Project No. 278417, and support in Pittsburgh from NSF Grant PHY-1148957. 

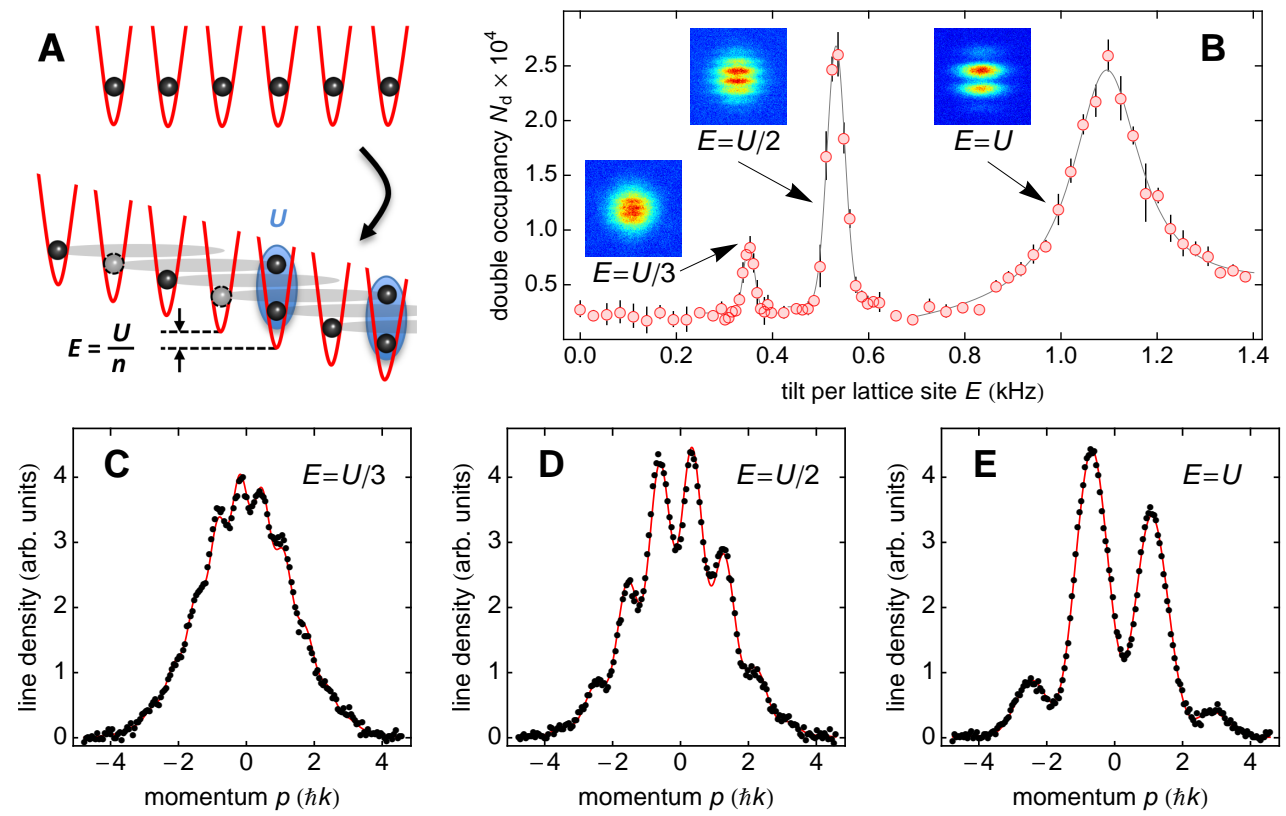

Fig. 1. Tunneling resonances in a tilted 1D Mott insulator. (A) Schematic view of the long-range correlations across $n$ sites for a tilt of $E=U / n$ after the quench from the initial 1D one-atom Mott insulator (top) to the tilted configuration (bottom). Here, $n=3$ for illustration purposes. (B) Number of doublons $N_{\mathrm{d}}$ as a function of $E$ for $t_{\mathrm{h}}=200 \mathrm{~ms}$ after the quench. Here $V_{z}=10 E_{\mathrm{R}}$ and $a_{\mathrm{s}}=252(5) \mathrm{a}_{0}$, giving $U=1.077(20) \mathrm{kHz}$ for $V_{x, y}=20 E_{\mathrm{R}}$. The solid lines are Lorentzian (for $E=U$ ) and Gaussian (for $E=U / 2$ and $E=U / 3$ ) fits to the data to determine the center positions and widths. The insets show matter-wave interference patterns obtained in TOF at $E_{1}=U, E_{2}=U / 2$, and $E_{3}=U / 3$ taken after $t_{\mathrm{h}}=1 \mathrm{~ms}, 9 \mathrm{~ms}$, and $28 \mathrm{~ms}$, respectively. (C)-(E) Integrated line densities of the TOF images shown in the insets in $(\mathbf{B})$. The solid lines are fits according to double slit interference patterns with Gaussian envelopes (29). Error bars in this and all other figures reflect the one-sigma standard deviation. 

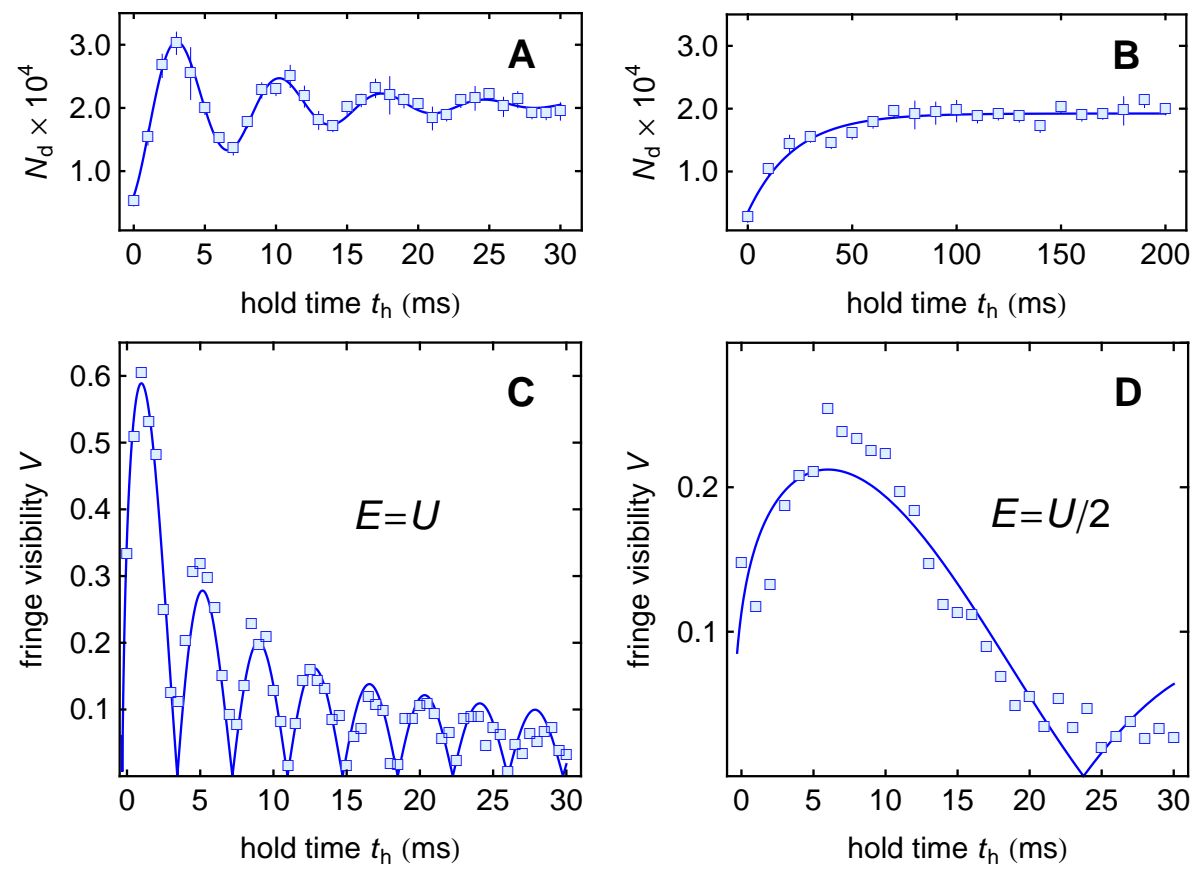

Fig. 2. Comparison of the tunneling dynamics to nearest and second-nearest neigh-

bors. Double occupancy $N_{\mathrm{d}},(\mathbf{A})$ and $(\mathbf{B})$, and fringe visibility $V$ in the TOF images, $(\mathbf{C})$ and (D), as a function of hold time $t_{\mathrm{h}}$ after the quench. Coherent oscillations in $N_{\mathrm{d}}$ at $E_{1}=U$ in (A) are contrasted to overdamped dynamics at $E_{2}=U / 2(\mathbf{B})$ for $V_{z}=10 E_{\mathrm{R}}$ and $a_{\mathrm{s}}=253(5) \mathrm{a}_{0}$. The evolution of $N_{\mathrm{d}}$ is fitted by an exponentially damped sinusoid and a saturated growth, respectively. The solid lines in (C) and (D) are fits to guide the eye based on the modulus of an algebraically decaying sinusoid. 

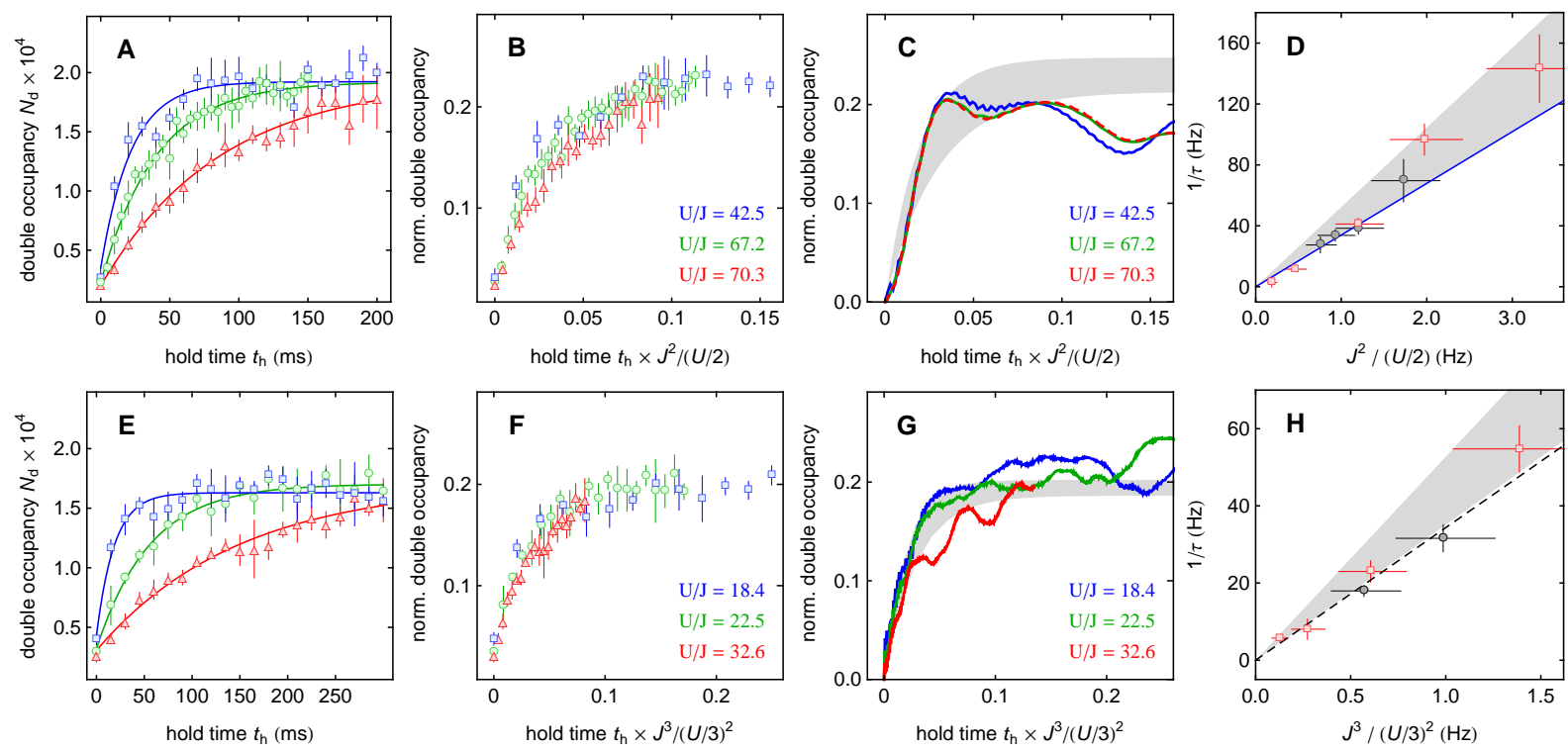

Fig. 3. Second- and third-order tunneling dynamics. Dependence of $N_{\mathrm{d}}$ on $J$ and $U$ at $E_{2}=U / 2,(\mathbf{A})-(\mathbf{D})$, and $E_{3}=U / 3,(\mathbf{E})-(\mathbf{H})$. (A) Double occupancy at $E_{2}$ for $\left(V_{z} / E_{\mathrm{R}}, a_{\mathrm{s}} / \mathrm{a}_{0}\right)$ $=(10,253(5))$ (squares), $(12,253(5))$ (triangles), and $(10,400(5))$ (circles). (E) Double occupancy at $E_{3}$ for $\left(V_{z} / E_{\mathrm{R}}, a_{\mathrm{s}} / \mathrm{a}_{0}\right)=(7,253(5))$ (squares), $(9,253(5))$ (triangles), and $(9,175(5))$ (circles). The solid lines are fits to the data with saturated growth functions. (B), (F) Collapse of the data shown in $(\mathbf{A})$ and $(\mathbf{E})$ for rescaled time axes. $(\mathbf{C}),(\mathbf{G})$ Result of a numerical simulation of the resonant response at $E_{2}$ and $E_{3}$, respectively. (D), (H) Growth rates $1 / \tau$ for $E_{2}$ and $E_{3}$, respectively. In (D) the data for $V_{z}=(8,9,10,12,14) E_{\mathrm{R}}$ with fixed $a_{\mathrm{s}}=253 \mathrm{a}_{0}$ (squares) and $a_{\mathrm{s}}=(175,253,325,400) \mathrm{a}_{0}$ with fixed $V_{z}=10 E_{\mathrm{R}}$ (circles) is plotted as a function of $J^{2} /(U / 2)$. The solid line gives the prediction from a three-site $\mathrm{BH}$ model (see text). In (H) the data for $V_{z}=(7,8,9,10) E_{\mathrm{R}}$ with $a_{\mathrm{s}}=253 \mathrm{a}_{0}$ (squares) and for $a_{\mathrm{s}}=175 \mathrm{a}_{0}$ at $V_{z}=9 E_{\mathrm{R}}$ and $a_{\mathrm{s}}=300 \mathrm{a}_{0}$ at $V_{z}=7 E_{\mathrm{R}}$ (circles) is plotted as a function of $J^{3} /(U / 3)^{2}$. The dashed line is a linear fit to the experimental data. The shaded areas in $(\mathbf{C}),(\mathbf{D}),(\mathbf{G})$, and $(\mathbf{H})$ indicate the spread in the growth rate extracted from the numerical data with fixed steady-state values from the experiment (22). 

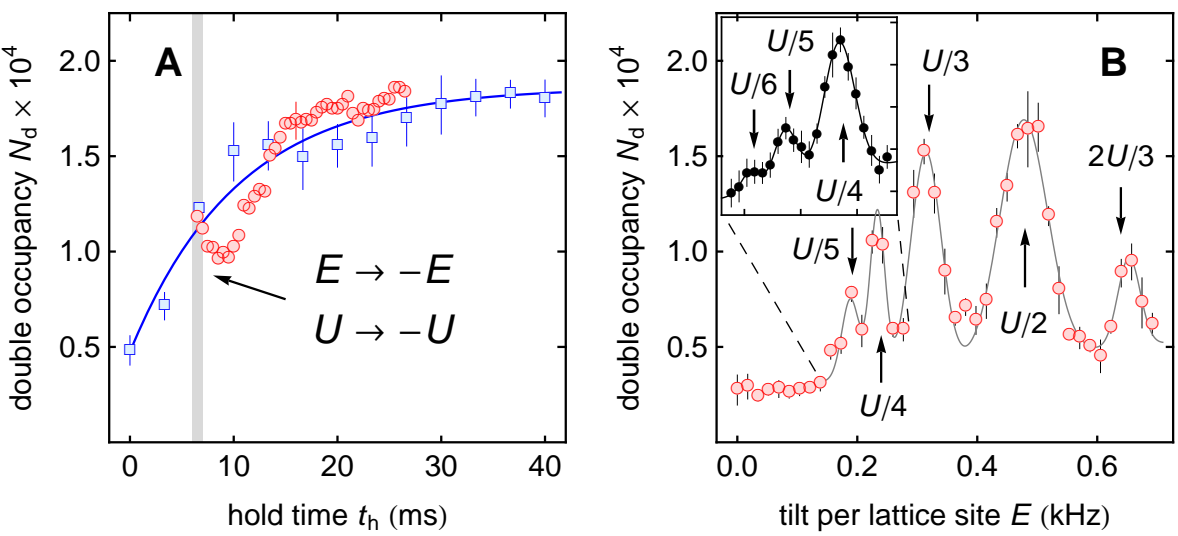

Fig. 4. Many-body echo and higher-order tunneling resonances. (A) Double occupancy $N_{\mathrm{d}}$ as a function of hold time $t_{\mathrm{h}}$ at $E=U / 2$ for $V_{z}=8 E_{\mathrm{R}}$ and $a_{\mathrm{s}}=-250(5) a_{0}$, giving $U=-994(20) \mathrm{Hz}$ (squares). Partial time reversal of the many-body dynamics (circles) after switching $a_{\mathrm{s}}$ to $+250(5) a_{0}$ and simultaneously reversing $E$ to $-E$ at $t_{\mathrm{h}}=6 \mathrm{~ms}$ within $1 \mathrm{~ms}$ (grey bar). For the echo data (circles) a typical error bar is given for a data point at $t_{\mathrm{h}}=16$ ms. (B) $N_{\mathrm{d}}$ as a function of $E$ after $t_{\mathrm{h}}=200 \mathrm{~ms}$ at $V_{z}=7 E_{\mathrm{R}}$ with $U=959(20) \mathrm{Hz}$, for $a_{\mathrm{s}}=252(5) \mathrm{a}_{0}$. The arrows indicate the expected positions of the tunneling resonances at $E_{n}=U / n$. An additional resonance at $2 U / 3$ appears. The inset gives a fine scan of the $U / 4$ and $U / 5$ resonances. The solid line is a fit based on the sum of multiple Gaussians to guide the eye. 